\title{
ON FUNCTIONAL GRAPHS OF QUADRATIC POLYNOMIALS
}

\author{
BERNARD MANS, MIN SHA, IGOR E. SHPARLINSKI, \\ AND DANIEL SUTANTYO
}

\begin{abstract}
We study functional graphs generated by quadratic polynomials over prime fields. We introduce efficient algorithms for methodical computations and provide the values of various direct and cumulative statistical parameters of interest. These include: the number of connected functional graphs, the number of graphs having a maximal cycle, the number of cycles of fixed size, the number of components of fixed size, as well as the shape of trees extracted from functional graphs. We particularly focus on connected functional graphs, that is, the graphs which contain only one component (and thus only one cycle). Based on the results of our computations, we formulate several conjectures highlighting the similarities and differences between these functional graphs and random mappings.
\end{abstract}

\section{INTRODUCTION}

Let $\mathbb{F}_{q}$ be the finite field of $q$ elements and of characteristic $p$, with $p \geqslant 3$. For a function $f: \mathbb{F}_{q} \rightarrow \mathbb{F}_{q}$, we define the functional graph of $f$ as a directed graph $\mathcal{G}_{f}$ on $q$ nodes labelled by the elements of $\mathbb{F}_{q}$ where there is an edge from $u$ to $v$ if and only if $f(u)=v$. For any integer $n \geqslant 1$, let $f^{(n)}$ be the $n$-th iteration of $f$.

These graphs are particular as one can immediately observe that each connected component of the graph $\mathcal{G}_{f}$ has a unique cycle (we treat fixed points as cycles of length 1$)$. An example for the functional graph of $x^{2}+12(\bmod 31)$ is given in Figure 1.1.

Recently, there have been an increasing interest in studying, theoretically and experimentally, the graphs $\mathcal{G}_{f}$ generated by polynomials $f \in \mathbb{F}_{q}[X]$ of small degree (such as quadratic polynomials), and how they differ, or not, from random mappings [Flajolet and Odlyzko 1990]. We refer to [Bellah et al. 2016, Bridy and Garton 2016, Burnette and

2010 Mathematics Subject Classification. 05C20, 05C85, $11 \mathrm{~T} 24$.

Key words and phrases. Polynomial maps, functional graphs, finite fields, random maps, algorithms. 


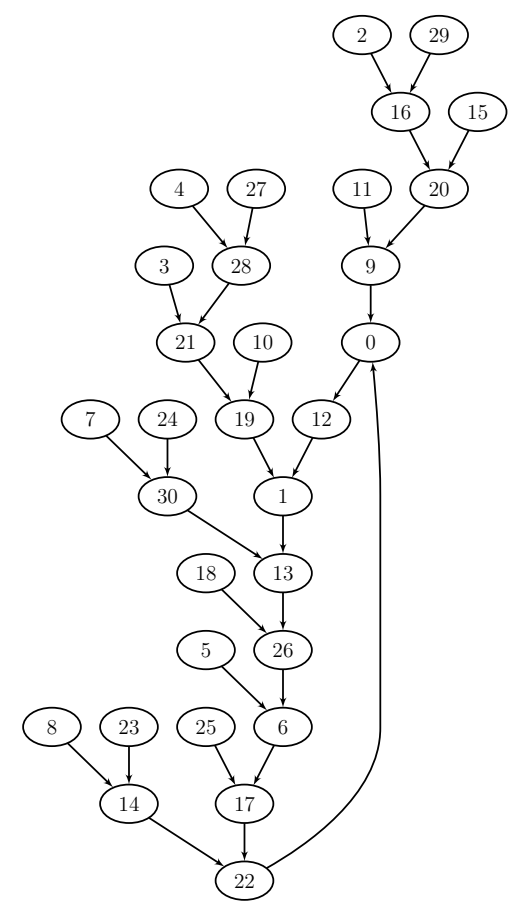

Figure 1.1. The functional graph of $X^{2}+12(\bmod 31)$

Schmutz 2017, Flynn and Garton 2014, Konyagin et al. 2016, Ostafe and Sha 2016] and the references therein.

In this paper, we concentrate on the case of quadratic polynomials over prime fields. In fact, up to isomorphism we only need to consider polynomials $f_{a}(X)=X^{2}+a, a \in \mathbb{F}_{p}$ (see the proof of [Konyagin et al. 2016, Theorem 2.1]). For simplicity, we use $\mathcal{G}_{a}=\mathcal{G}_{f_{a}}$ to denote the functional graph generated by $f_{a}$. For this case, in [Konyagin et al. 2016, Section 4] the authors have provided numerical data for the number of distinct graphs $\mathcal{G}_{a}$, the statistics of cyclic points, the number of connected components, as well as the most popular component size.

Different from the aspects in [Konyagin et al. 2016], we consider several questions related to distributions of cyclic points and sizes of connected components of $\mathcal{G}_{a}$ when $a$ runs through the elements in $\mathbb{F}_{p}$. In particular, we are interested in characterising connected functional graphs $\mathcal{G}_{a}$, that is, the graphs which contain only one component (and thus only one cycle).

In this paper, we focus on characterising the functional graphs by providing direct parameters such as the number of (connected) components. We then characterise various cumulative parameters, such as the number of cyclic points and the shape of trees extracted from 
functional graphs. We highlight similarities and differences between functional graphs [Konyagin et al. 2016] and random mappings [Flajolet and Odlyzko 1990], and we also pay much attention to features of connected functional graphs. While obtaining theoretic results for these questions remains a challenge, we introduce efficient algorithms and present new interesting results of numerical experiments.

The rest of the paper is structured as follows. In Section 2, we develop a fast algorithm that determines whether a functional graph is connected, which is used to compute the number of connected functional graphs. In Section 3, we compare the number of cyclic points in connected graphs with those in all graphs modulo $p$. In Section 4 and Section 5 respectively, we consider the number of components with small number of cyclic points and with small size. Finally, in Section 6 we illustrate the statistics of trees in functional graphs.

Throughout the paper, we use the Landau symbol $O$. Recall that the assertion $U=O(V)$ is equivalent to the inequality $|U| \leqslant c V$ with some absolute constant $c>0$. To emphasise the dependence of the implied constant $c$ on some parameter (or a list of parameters) $\rho$, we write $U=O_{\rho}(V)$. We also use the asymptotic symbol $\sim$.

\section{Counting COnneCted GRAPhs}

In this section, we introduce a new efficient algorithm that quickly detects connected functional graphs, and formulate some conjectures for the number of connected graphs based on our computations.

2.1. Preliminaries and informal ideas of the algorithm. Let $\mathcal{I}_{p}$ be the set $a \in \mathbb{F}_{p}$ such that $\mathcal{G}_{a}$ is connected. We also denote by $I_{p}=\# \mathcal{I}_{p}$ the number of connected graphs $\mathcal{G}_{a}$ with $a \in \mathbb{F}_{p}$. Clearly the graph $\mathcal{G}_{0}$ is not connected, and also by [Vasiga and Shallit 2004, Corollary 18 (a)] $\mathcal{G}_{-2}$ is also not connected if $p>3$, and so $\mathcal{I}_{p} \subseteq \mathbb{F}_{p} \backslash\{0,-2\}$ if $p>3$. In fact, the functional graphs with values $a=0$ and $a=-2$ lead to graphs with a particular group structure (and thus the structure of these graphs deviates significantly from the other graphs, see [Vasiga and Shallit 2004]).

Essentially in [Konyagin et al. 2016, Algorithm 3.1], a rigorous deterministic algorithm using Floyd's cycle detection algorithm and needing $O(p)$ function evaluations (that is, of complexity $p^{1+o(1)}$ ) has been used to test whether $\mathcal{G}_{a}$ is a connected graph. Instead of evaluating $I_{p}$ via this algorithm which would need $O\left(p^{2}\right)$ function evaluations, we introduce a more efficient heuristic approach in practice, which is specifically useful for computations of a family of graphs (not just a single graph). 
The main idea is to first check quickly whether $\mathcal{G}_{a}$ has more than one small cycle (i.e., more than one component). A graph $\mathcal{G}_{a}$ has a component with a cycle of size $\ell$ if and only if the equation $f_{a}^{(\ell)}(u)=$ $u$ has a solution $u$ which is not a solution to any of the equations $f_{a}^{(k)}(u)=u$ with $1 \leqslant k<\ell$. The roots of $f_{a}^{(\ell)}(u)=u$ are the cyclic points in the graph. For this we need the dynatomic polynomials

$$
F_{a}^{(\ell)}(X)=\prod_{r \mid \ell}\left(f_{a}^{(r)}(X)-X\right)^{\mu(\ell / r)},
$$

where $\mu(k)$ is the Möbius function, see [Silverman 2007, Section 4.1]. Moreover, we have

$$
f_{a}^{(n)}(X)-X=\prod_{\ell \mid n} F_{a}^{(\ell)}(X), \quad n=1,2, \ldots
$$

For example

$$
F_{a}^{(1)}(X)=X^{2}-X+a \text { and } F_{a}^{(2)}(X)=X^{2}+X+a+1
$$

and

$$
F_{a}^{(3)}(X)=\left(f_{a}^{(3)}(X)-X\right) /\left(f_{a}^{(1)}(X)-X\right) .
$$

Clearly, if $\mathcal{G}_{a}$ has a cycle of length $\ell$, then any point in this cycle is a root of the polynomial $F_{a}^{(\ell)}(X)$. However, the roots of $F_{a}^{(\ell)}(X)$ might be not all lying in cycles of length $\ell$; for instance see [Silverman 2007, Example 4.2]. Certainly, $\mathcal{G}_{a}$ is not connected if $F_{a}^{(\ell)}(X)$ has a root for two distinct values of $\ell=\ell_{1}, \ell_{2}$ with $\ell_{1} \nmid \ell_{2}$ and $\ell_{2} \nmid \ell_{1}$. Alternatively, if $F_{a}^{(\ell)}(X)$ has more than $\ell$ distinct roots, this indicates that $\mathcal{G}_{a}$ has at least two cycles, which again implies that $\mathcal{G}_{a}$ has more than one connected component.

As we show later, it turns out that this occurs frequently and thus we can rule out the connectivity of most of the graph $\mathcal{G}_{a}, a \in \mathbb{F}_{p}$ quickly. A relatively small number of remaining suspects can be checked via the rigorous deterministic algorithm from [Konyagin et al. 2016, Algorithm 3.1 .

2.2. Algorithm. Algorithm 2.1 is to determine whether a graph is connected or not, where we in fact use $f_{a}^{(\ell)}(X)$ instead of $F_{a}^{(\ell)}(X)$.

The algorithm starts by checking if there is any cycle of size 1 in the graph. Since $X^{p}-X$ only contains simple roots and $f_{a}^{(1)}(X)$ has degree 2 , if $\operatorname{gcd}\left(X^{p}-X, f_{a}^{(1)}(X)\right)>1$, then there are two cycles of size 1 and thus two separate components in the graph. Otherwise, there is at most one component with a cycle of size 1 in the graph $\mathcal{G}_{a}$. 


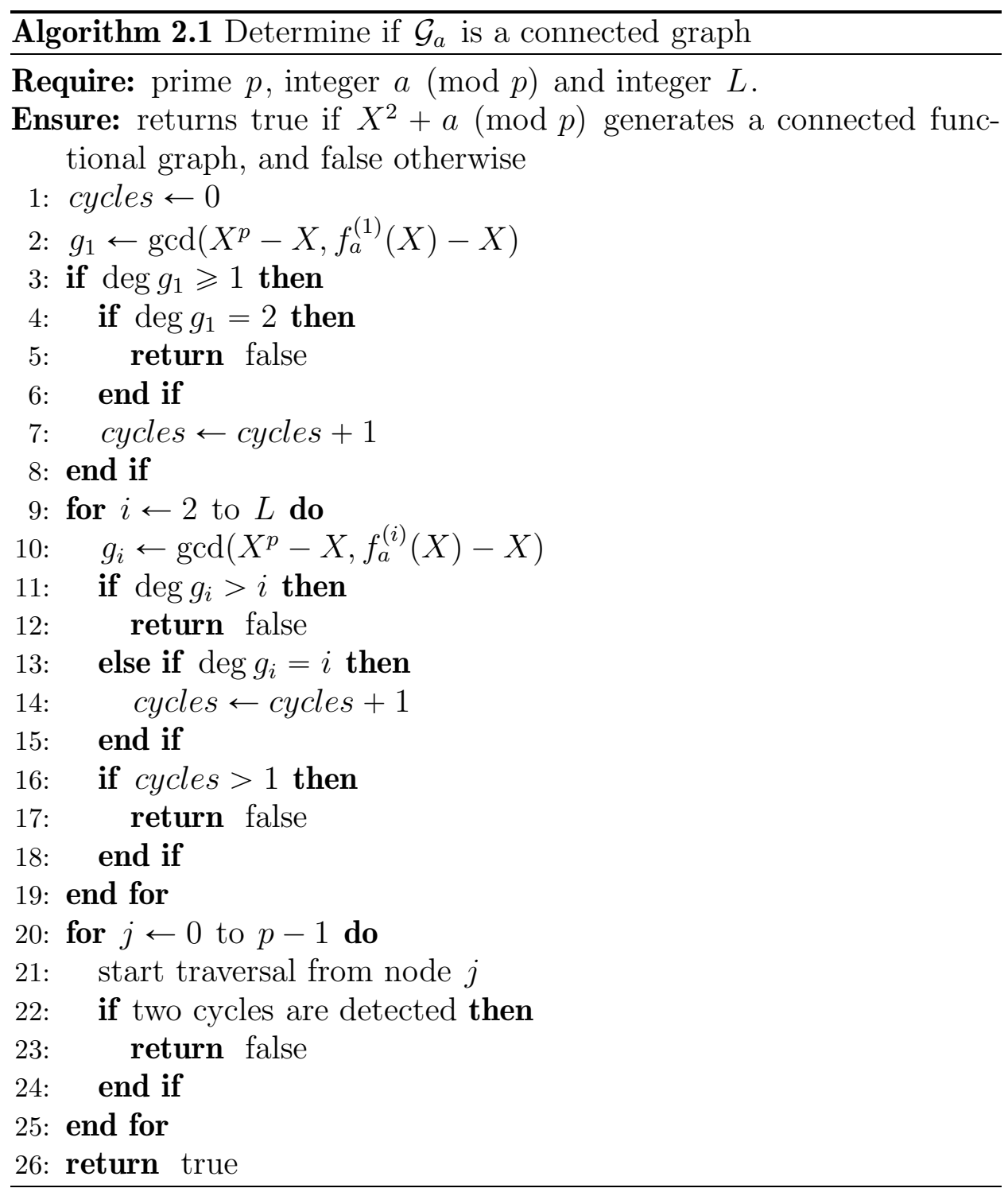

Next, we compute $g_{i}=\operatorname{gcd}\left(X^{p}-X, f_{a}^{(i)}(X)-X\right)$ from $i=2$ until $L$ while keeping track of the number of cycles that has been detected. Here, we have several possibilities:

- if $\operatorname{deg} g_{i}<i$, then there are no cycle of size $i$ in the graph.

- if $\operatorname{deg} g_{i}=i$, then there is exactly one cycle of size $i$.

- if $\operatorname{deg} g_{i}>i$, then there are at least two different cycles in the graph.

When $\operatorname{deg} g_{i}<i$, there are no cycle of size $i$ since there are not enough roots to form one. Similarly, if $\operatorname{deg} g_{i}>i$, then there are more 
than $i$ cyclic points in the graph, of which at least $i$ of them form one cycle, and so there are more than one cycle in the graph.

Finally, if at this stage the algorithm detects $\operatorname{deg} g_{i}=i$, then there is exactly one cycle of size $i$. By contradiction, if there is no cycle of size $i$, then there must be at least two cycles of size less than $i$, and so we would have detected that cycles $>1$ at a previous iteration, thus returning 'false'.

Once we are done with the first loop, either we have found one cycle with size at most $L$, or we have not found any small cycles at all. We then proceed with a graph traversal until we find two cycles.

2.3. Statistics of the number of connected graphs. We implement Algorithm 2.1 by using NTL [NTL 2016] and PARI/GP [Pari 2016], choosing $L=5$ in our computations. We collect values of $I_{p}$ for some primes (as shown in Table 2.1) that lead us to the following conjecture:

Conjecture 2.1. $I_{p} \sim \sqrt{2 p}$ as $p \rightarrow \infty$.

Here, we also pose a weaker conjecture:

Conjecture 2.2. For any prime $p, I_{p} \geqslant 1$.

Conjecture 2.2 predicts that there always exists a connected functional graph generated by quadratic polynomials modulo $p$. Indeed, according to our computations, Conjecture 2.2 is true for all primes $p \leqslant 100000$. 


\begin{tabular}{crc}
\hline$p$ & \multicolumn{1}{c}{$I_{p}$} & $\sqrt{2 p}$ \\
\hline 500,009 & 1,038 & $1,000.009$ \\
500,029 & 1,002 & $1,000.029$ \\
500,041 & 956 & $1,000.041$ \\
500,057 & 1,026 & $1,000.057$ \\
500,069 & 995 & $1,000.069$ \\
500,083 & 987 & $1,000.083$ \\
500,107 & 994 & $1,000.107$ \\
500,111 & 1,010 & $1,000.111$ \\
500,113 & 1,019 & $1,000.113$ \\
500,119 & 920 & $1,000.119$ \\
500,153 & 1,033 & $1,000.153$ \\
500,167 & 1,005 & $1,000.167$ \\
$1,000,003$ & 1,369 & $1,414.296$ \\
$2,000,003$ & 1,909 & $2,000.001$ \\
$3,000,017$ & 2,478 & $2,449.497$ \\
$4,000,037$ & 2,838 & $2,828.440$ \\
\hline
\end{tabular}

TABLE 2.1. The number of connected graphs modulo $p$

We also investigate the existence of connected functional graphs having (only) one cycle of size 1.

If the graph $\mathcal{G}_{a}$ is connected and has one cycle of size 1 , then the equation $X^{2}+a=X$ has two identical roots (corresponding to fixed points), and so $a=1 / 4$ and the root $x=1 / 2$. Thus, we only need to check the graph generated by $X^{2}+1 / 4$ in $\mathbb{F}_{p}$.

We have tested all the primes up to 100000 and we only have found two such examples: one is $X^{2}+1$ in $\mathbb{F}_{3}$, and the other is $X^{2}+2$ in $\mathbb{F}_{7}$. Furthermore, we have:

Proposition 2.3. For any prime $p$ with $p \equiv 5$ or $11(\bmod 12)$, there is no functional graph $\mathcal{G}_{a}$ having only one cycle of size 1 .

Proof. Note that we only need to consider the graph $\mathcal{G}_{1 / 4}$. Since $1 / 2$ is a fixed point of $\mathcal{G}_{1 / 4}$ and there is an edge from $-1 / 2$ to $1 / 2$, we consider the equation $X^{2}+1 / 4=-1 / 2$ in $\mathbb{F}_{p}$, that is, whether -3 is a square in $\mathbb{F}_{p}$. However, if $p \equiv 5$ or $11(\bmod 12),-3$ is not a square in $\mathbb{F}_{p}$. Then, the in-degree of $-1 / 2$ is zero, and so $\mathcal{G}_{1 / 4}$ must have more than one cycle. This completes the proof.

So, we pose the following conjecture:

Conjecture 2.4. For any prime $p>7$, there is no functional graph $\mathcal{G}_{a}$ having only one cycle of size 1 . 


\section{Counting CYClic POINTS In FUnCtional GRAPhS}

We now assess the number of cyclic points in functional graphs modulo $p$. For the minimal and maximal numbers of cyclic points in graphs $\mathcal{G}_{a}$, we refer to [Konyagin et al. 2016, Table 4.1], where the cases $a=0,-2$ are excluded. Roughly speaking, the reason why these two cases are excluded is that the number of cyclic points is maximized on the cases $a=0,-2$ quite often; see [Konyagin et al. 2016, Section 4.3] for more details. In this section, we also follow this convention.

Let $C_{a}$ be the total number of cyclic points of $\mathcal{G}_{a}$, and let $c_{a}$ be the largest number of cyclic points in a single component of $\mathcal{G}_{a}$. Clearly we have $C_{a} \geqslant c_{a}$ for any $a \in \mathbb{F}_{p}$ and $C_{a}=c_{a}$ when $a \in \mathcal{I}_{p}$.

Furthermore, we define the average and largest values of these quantities:

$$
\begin{array}{ll}
\overline{C_{p}}=\frac{1}{p-2} \sum_{a \in \mathbb{F}_{p} \backslash\{0,-2\}} C_{a}, & \mathbf{C}_{p}=\max \left\{C_{a}: a \in \mathbb{F}_{p} \backslash\{0,-2\}\right\} ; \\
\overline{c_{p}}=\frac{1}{p-2} \sum_{a \in \mathbb{F}_{p} \backslash\{0,-2\}} c_{a}, & \mathbf{c}_{p}=\max \left\{c_{a}: a \in \mathbb{F}_{p} \backslash\{0,-2\}\right\} ; \\
{\overline{c_{p}}}^{*}=\frac{1}{I_{p}} \sum_{a \in \mathcal{I}_{p}} c_{a}, & \mathbf{c}_{p}^{*}=\max \left\{c_{a}: a \in \mathcal{I}_{p}\right\} .
\end{array}
$$

We remark again that $\mathcal{I}_{p} \subseteq \mathbb{F}_{p} \backslash\{0,-2\}$ if $p>3$.

Numerical experiments in [Konyagin et al. 2016, Section 4.3] suggest that the average number of cyclic points modulo $p$, taken over all graphs modulo $p$ (excluding $a=0,-2$ ), is $\sqrt{\pi p / 2}$, which is consistent with the behaviour of random maps (see [Flajolet and Odlyzko 1990, Theorem 2(ii)]). Here we show that this is not the case for connected graphs (see Table 3.1 ). In that case, ${\overline{c_{p}}}^{*}$ is smaller than $\overline{C_{p}}$, i.e. there are fewer cyclic points than those for non-connected graphs on average. Notice that both ${\overline{c_{p}}}^{*}$ and $\overline{c_{p}}$ are both close to $\sqrt{2 p / \pi}$ (and although close to each other, ${\overline{c_{p}}}^{*}$ is slightly larger). 


\begin{tabular}{rrrrrr}
\hline \multicolumn{1}{c}{$p$} & \multicolumn{1}{c}{$\overline{C_{p}}$} & $\sqrt{\pi p / 2}$ & \multicolumn{1}{c}{$\overline{c_{p}}$} & \multicolumn{1}{c}{$\bar{c}_{p}^{*}$} & $\sqrt{2 p / \pi}$ \\
\hline 500,009 & 886.224 & 886.235 & 553.445 & 573.355 & 564.194 \\
500,029 & 885.990 & 886.253 & 553.312 & 587.750 & 564.205 \\
500,041 & 885.069 & 886.263 & 553.175 & 568.208 & 564.212 \\
500,057 & 884.963 & 886.277 & 552.870 & 586.037 & 564.221 \\
500,069 & 885.831 & 886.288 & 552.952 & 558.285 & 564.229 \\
500,083 & 884.970 & 886.300 & 552.692 & 564.995 & 564.236 \\
500,107 & 884.507 & 886.322 & 552.674 & 562.690 & 564.250 \\
500,111 & 884.341 & 886.325 & 552.157 & 575.976 & 564.252 \\
500,113 & 885.160 & 886.327 & 552.988 & 568.057 & 564.253 \\
500,119 & 884.559 & 886.332 & 552.597 & 569.750 & 564.257 \\
500,153 & 884.834 & 886.363 & 552.900 & 589.146 & 564.276 \\
500,167 & 885.756 & 886.375 & 552.525 & 560.095 & 564.284 \\
600,011 & 969.139 & 970.822 & 605.632 & 611.914 & 618.044 \\
700,001 & $1,047.771$ & $1,048.599$ & 654.317 & 667.624 & 667.559 \\
800,011 & $1,120.427$ & $1,121.006$ & 700.047 & 703.061 & 713.655 \\
900,001 & $1,188.822$ & $1,188.999$ & 742.619 & 762.673 & 756.940 \\
$1,000,003$ & $1,252.452$ & $1,253.316$ & 782.026 & 793.388 & 797.886 \\
$2,000,003$ & $1,772.078$ & $1,772.455$ & $1,106.815$ & $1,134.598$ & $1,128.380$ \\
\hline
\end{tabular}

TABLE 3.1. Average number of cyclic points in graphs modulo $p$ (excluding $a=0,-2$ )

In Table 3.2, one can see that the largest cycles usually do not appear in the connected graphs, which appears surprising and shows the existence of components with a large cycle even when the graph is disconnected. In addition, the difference $\mathbf{c}_{p}-\mathbf{c}_{p}^{*}$ is large, while the difference of $\mathbf{C}_{p}$ and $\mathbf{c}_{p}$ is small. 


\begin{tabular}{rccc}
\hline \multicolumn{1}{c}{$p$} & $\mathbf{C}_{p}$ & $\mathbf{c}_{p}$ & $\mathbf{c}_{p}^{*}$ \\
\hline 500,009 & 3,578 & 3,164 & 2,319 \\
500,029 & 3,620 & 3,291 & 2,327 \\
500,041 & 3,798 & 3,118 & 2,333 \\
500,057 & 3,468 & 3,319 & 2,423 \\
500,069 & 3,556 & 3,129 & 2,089 \\
500,083 & 3,596 & 3,050 & 2,131 \\
500,107 & 3,527 & 3,232 & 2,643 \\
500,111 & 3,732 & 3,237 & 2,244 \\
500,113 & 3,805 & 3,232 & 2,335 \\
500,119 & 3,873 & 3,142 & 2,275 \\
500,153 & 3,472 & 3,380 & 2,754 \\
500,167 & 3,644 & 3,159 & 2,770 \\
600,011 & 3,847 & 3,488 & 3,265 \\
700,001 & 4,350 & 3,670 & 2,950 \\
800,011 & 4,600 & 4,242 & 3,208 \\
900,001 & 4,997 & 4,274 & 3,245 \\
$1,000,003$ & 5,101 & 4,639 & 3,117 \\
$2,000,003$ & 7,637 & 6,848 & 4,309 \\
\hline
\end{tabular}

TABLE 3.2. Maximum number of cyclic points in graphs modulo $p$ (excluding $a=0,-2$ )

Let us also define the following three families of parameters $a$ on which the values $\mathbf{C}_{p}, \mathbf{c}_{p}$ and $\mathbf{c}_{p}^{*}$ are achieved, that is

$$
\begin{aligned}
& \mathscr{A}_{p}=\left\{a \in \mathbb{F}_{p} \backslash\{0,-2\}: C_{a}=\mathbf{C}_{p}\right\}, \\
& \mathscr{B}_{p}=\left\{a \in \mathbb{F}_{p} \backslash\{0,-2\}: c_{a}=\mathbf{c}_{p}\right\}, \\
& \mathscr{B}_{p}^{*}=\left\{a \in \mathcal{I}_{p}: c_{a}=\mathbf{c}_{p}^{*}\right\} .
\end{aligned}
$$

It is certainly interesting to compare the sizes $A_{p}=\# \mathscr{A}_{p}, B_{p}=\# \mathscr{B}_{p}$ and $B_{p}^{*}=\# \mathscr{B}_{p}^{*}$ and also investigate the mutual intersections between these families.

We find that typically these sets have one value of $a$ in common, and rarely more than two. As $p$ increases, the frequency of the sets having 2 or more elements decreases, but does not disappear completely, as can be seen in Table 3.3. 


\begin{tabular}{l|rrrrrrrrrr}
\hline & \multicolumn{3}{|c}{$A_{p}$} & \multicolumn{3}{c}{$B_{p}$} & \multicolumn{3}{c}{$B_{p}^{*}$} \\
range of $p$ & $=1$ & $=2$ & $\geqslant 3$ & $=1$ & $=2$ & $=3$ & $=1$ & $=2$ & $\geqslant 3$ \\
\hline$\left[3,10^{4}\right]$ & 1,182 & 39 & 7 & 1,159 & 65 & 4 & 1,193 & 35 & 0 \\
{$\left[10^{4}, 2 \cdot 10^{4}\right]$} & 1,013 & 20 & 0 & 1,010 & 22 & 1 & 1,019 & 14 & 0 \\
{$\left[2 \cdot 10^{4}, 3 \cdot 10^{4}\right]$} & 967 & 14 & 2 & 970 & 13 & 0 & 976 & 7 & 0 \\
{$\left[3 \cdot 10^{4}, 4 \cdot 10^{4}\right]$} & 949 & 9 & 0 & 941 & 17 & 0 & 950 & 8 & 0 \\
{$\left[4 \cdot 10^{4}, 5 \cdot 10^{4}\right]$} & 921 & 8 & 1 & 921 & 9 & 0 & 926 & 4 & 0 \\
{$\left[5 \cdot 10^{4}, 6 \cdot 10^{4}\right]$} & 915 & 9 & 0 & 920 & 4 & 0 & 921 & 3 & 0 \\
{$\left[6 \cdot 10^{4}, 7 \cdot 10^{4}\right]$} & 868 & 10 & 0 & 872 & 6 & 0 & 868 & 9 & 1 \\
{$\left[7 \cdot 10^{4}, 8 \cdot 10^{4}\right]$} & 895 & 7 & 0 & 897 & 5 & 0 & 899 & 3 & 0 \\
{$\left[8 \cdot 10^{4}, 9 \cdot 10^{4}\right]$} & 869 & 7 & 0 & 869 & 7 & 0 & 866 & 10 & 0 \\
{$\left[9 \cdot 10^{4}, 10^{5}\right]$} & 874 & 5 & 0 & 878 & 1 & 0 & 876 & 3 & 0 \\
{$\left[10^{5}, 10^{5}+10^{3}\right]$} & 81 & 0 & 0 & 79 & 2 & 0 & 81 & 0 & 0 \\
{$\left[10^{6}, 10^{6}+10^{3}\right]$} & 74 & 1 & 0 & 75 & 0 & 0 & 74 & 1 & 0 \\
\hline & \multicolumn{10}{c}{}
\end{tabular}

TABLE 3.3. Values of $A_{p}, B_{p}$, and $B_{p}^{*}$

For the set intersections, we start with $\mathscr{A}_{p} \cap \mathscr{B}_{p}^{*}$. With Table 3.1, we have observed that $\overline{C_{p}}>\overline{c_{p}}$ * , thus it is reasonable to expect that $\mathscr{A}_{p} \cap \mathscr{B}_{p}^{*}$ is empty. We remark that if $\mathscr{A}_{p} \cap \mathscr{B}_{p}^{*}$ is not empty, then $\mathbf{C}_{p}=\mathbf{c}_{p}=\mathbf{c}_{p}^{*}$, and so for any $a \in \mathscr{B}_{p}$ the graph $\mathcal{G}_{a}$ is connected, and thus $\mathscr{B}_{p}=\mathscr{B}_{p}^{*}$. Therefore, for any prime $p$, if $\mathbf{c}_{p}<\mathbf{C}_{p}$, then we must have that $\mathscr{A}_{p} \cap \mathscr{B}_{p}^{*}$ is empty. Our experiments with odd prime $p<10^{5}$ counted only 20 occurrences of primes where the intersection is non-empty and in fact contains only one value of $a$, shown in Table 3.4.

\begin{tabular}{cccc}
\hline$p$ & value of $a$ & $p$ & value of $a$ \\
\hline 3 & 2 & 271 & 147 \\
5 & 1 & 2,647 & 1,445 \\
7 & 3 & 3,613 & 2,653 \\
11 & 6 & 6,131 & 3,555 \\
13 & 1 & 6,719 & 107 \\
17 & 3 & 17,921 & 8,370 \\
19 & 13 & 18,077 & 15,557 \\
29 & 4 & 36,229 & 2,229 \\
157 & 141 & 53,611 & 23,630 \\
191 & 97 & 64,667 & 60,638 \\
\hline
\end{tabular}

TABLE 3.4. Values of $p$ with non-empty $\mathscr{A}_{p} \cap \mathscr{B}_{p}^{*}$ 
Since we have observed only one value of $a$ for each prime $p$ in the above table, we conjecture that:

Conjecture 3.1. For any prime $p \geqslant 3$, we have $\#\left(\mathscr{A}_{p} \cap \mathscr{B}_{p}^{*}\right) \leqslant 1$.

We also consider the intersection $\mathscr{B}_{p} \cap \mathscr{B}_{p}^{*}$; see Table 3.5. Clearly, if $\mathscr{B}_{p} \cap \mathscr{B}_{p}^{*}$ is not empty, then we have $\mathbf{c}_{p}=\mathbf{c}_{p}^{*}$. One could expect the number of primes with non-empty intersections to decrease as $p$ increases, however even if our experiments show some reduction overall, it remains unclear.

\begin{tabular}{lrrc}
\hline range of $p$ & freq & \#primes & $\%$ \\
\hline$\left[3,10^{4}\right]$ & 104 & 1,228 & $8.06 \%$ \\
{$\left[10^{4}, 2 \cdot 10^{4}\right]$} & 35 & 1,033 & $3.19 \%$ \\
{$\left[2 \cdot 10^{4}, 3 \cdot 10^{4}\right]$} & 32 & 983 & $3.26 \%$ \\
{$\left[3 \cdot 10^{4}, 4 \cdot 10^{4}\right]$} & 20 & 958 & $1.98 \%$ \\
{$\left[4 \cdot 10^{4}, 5 \cdot 10^{4}\right]$} & 19 & 930 & $2.04 \%$ \\
{$\left[5 \cdot 10^{4}, 6 \cdot 10^{4}\right]$} & 16 & 924 & $1.73 \%$ \\
{$\left[6 \cdot 10^{4}, 7 \cdot 10^{4}\right]$} & 20 & 878 & $2.28 \%$ \\
{$\left[7 \cdot 10^{4}, 8 \cdot 10^{4}\right]$} & 15 & 902 & $1.66 \%$ \\
{$\left[8 \cdot 10^{4}, 9 \cdot 10^{4}\right]$} & 15 & 876 & $1.71 \%$ \\
{$\left[9 \cdot 10^{4}, 10^{5}\right]$} & 6 & 879 & $0.68 \%$ \\
{$\left[10^{5}, 10^{5}+10^{3}\right]$} & 0 & 81 & $0.00 \%$ \\
{$\left[10^{6}, 10^{6}+10^{3}\right]$} & 1 & 75 & $1.33 \%$ \\
\hline
\end{tabular}

TABLE 3.5. Primes with non-empty $\mathscr{B}_{p} \cap \mathscr{B}_{p}^{*}$

The most surprising result comes from the observation of the intersection $\mathscr{A}_{p} \cap \mathscr{B}_{p}$. As Table 3.6 shows, the event that this intersection is not empty is rather common. For any $a \in \mathscr{A}_{p} \cap \mathscr{B}_{p}$, the graph $\mathcal{G}_{a}$ not only has the maximal number of cyclic points but also has a maximal cycle.

Note that for the last two rows we only give primes in the ranges $\left[10^{5}, 10^{5}+10^{3}\right]$ and $\left[10^{6}, 10^{6}+10^{3}\right]$, respectively, due to the limits of our current computational facilities. 


\begin{tabular}{lrrc}
\hline range of $p$ & freq & \#primes & $\%$ \\
\hline$\left[3,1 \cdot 10^{4}\right]$ & 268 & 1,228 & $20.36 \%$ \\
{$\left[10^{4}, 2 \cdot 10^{4}\right]$} & 197 & 1,033 & $18.87 \%$ \\
{$\left[2 \cdot 10^{4}, 3 \cdot 10^{4}\right]$} & 153 & 983 & $15.16 \%$ \\
{$\left[3 \cdot 10^{4}, 4 \cdot 10^{4}\right]$} & 148 & 958 & $15.24 \%$ \\
{$\left[4 \cdot 10^{4}, 5 \cdot 10^{4}\right]$} & 126 & 930 & $13.55 \%$ \\
{$\left[5 \cdot 10^{4}, 6 \cdot 10^{4}\right]$} & 167 & 924 & $17.97 \%$ \\
{$\left[6 \cdot 10^{4}, 7 \cdot 10^{4}\right]$} & 143 & 878 & $16.17 \%$ \\
{$\left[7 \cdot 10^{4}, 8 \cdot 10^{4}\right]$} & 143 & 902 & $15.74 \%$ \\
{$\left[8 \cdot 10^{4}, 9 \cdot 10^{4}\right]$} & 144 & 876 & $16.44 \%$ \\
{$\left[9 \cdot 10^{4}, 10^{5}\right]$} & 147 & 879 & $16.72 \%$ \\
{$\left[10^{5}, 10^{5}+10^{3}\right]$} & 13 & 81 & $16.05 \%$ \\
{$\left[10^{6}, 10^{6}+10^{3}\right]$} & 9 & 77 & $11.69 \%$ \\
\hline
\end{tabular}

TABle 3.6. Primes with non-empty $\mathscr{A}_{p} \cap \mathscr{B}_{p}$

\section{Statistics OF SMALL CYCLES}

We now study components by analysing the distribution of the size of their cycles. Let $\mathcal{C}_{a, k}$ be the number of cycles of length $k$ in the graph $\mathcal{G}_{a}$. Let

$$
\mathcal{C}_{k}=\sum_{a \in \mathbb{F}_{p}} \mathcal{C}_{a, k}
$$

be the number of cycles of length $k$ over all graphs modulo $p$. Clearly, we have $\mathcal{C}_{k}=0$ for any $k \geqslant p / 2$; see [Peinado et al. 2001, Theorems 1 and 2] for better bounds of $k$.

Proposition 4.1. For any integer $k \geqslant 1$, there is a constant $D_{k}$ depending only on $k$ such that for any prime $p>D_{k}$ we have

$$
\mathcal{C}_{k}=p / k+O\left(4^{k} k^{-1} p^{1 / 2}\right) .
$$

Proof. We can assume that $p>k$. For any fixed $a$, notice that any point $x$ contributing to $\mathcal{C}_{a, k}$ is a root of the polynomial $F_{a}^{(k)}(X)$. Conversely, any root $x$ of $F_{a}^{(k)}(X)$ contributes to $\mathcal{C}_{a, d}$ for some $d \mid k$ (possibly $d \neq k$ ). Thus, we have

$$
k \mathcal{C}_{k} \leqslant \#\left\{(a, x) \in \mathbb{F}_{p}^{2}: F_{a}^{(k)}(x)=0\right\} .
$$

Moreover, from [Morton and Patel 1994, Theorem 2.4 (c)] and noticing $p \nmid k$, we know that if $F_{a}^{(d)}(x)=0$ and $F_{a}^{(k)}(x)=0$ with $d<k$, where $x$ is a point lying in a cycle of length $k$, then $(X-x)^{2} \mid F_{a}^{(k)}(X)$, that is, the discriminant of $F_{a}^{(k)}(X)$ is zero. Note that as a polynomial in 
$X$ the degree of $F_{a}^{(k)}(X)$ is at most $2^{k}$, and as a polynomial in $a$ the degree of $F_{a}^{(k)}(X)$ is at most $2^{k-1}$. Then, as a polynomial in $a$, the degree of the discriminant of $F_{a}^{(k)}(X)$ is at most $4^{k}$. Thus, except for at most $4^{k}$ values of $a$, we have that $F_{a}^{(k)}(X)$ is a simple polynomial in $X$. Hence, we have

$$
k \mathcal{C}_{k}=\#\left\{(a, x) \in \mathbb{F}_{p}^{2}: F_{a}^{(k)}(x)=0\right\}+O\left(8^{k}\right) .
$$

In addition, combining [Morton 1996, Corollary 1 to Theorem B] with [Morton and Vivaldi 1995, Proposition 3.2], if we view $f_{A}(X)=$ $X^{2}+A$ as an integer polynomial in variables $A$ and $X$, then $F_{A}^{(k)}(X) \in$ $\mathbb{Z}[A, X]$ is an absolutely irreducible polynomial. Then, by Ostrowski's theorem, there exists a positive integer $D_{k}$ depending only on $k$ such that for any $p>D_{k}$ the polynomial $F_{A}^{(k)}(X)$ is absolutely irreducible modulo $p$ in variables $A$ and $X$. It is also easy to see by induction on $k$ that $f_{A}^{(k)}(X)$ is of total degree at most $2^{k}$ as a bivariate polynomial in $A$ and $X$, and the same is true for $F_{A}^{(k)}(X)$. Thus, by the Hasse-Weil bound (see [Lorenzini 1996, Section VIII.5.8]) we obtain

$$
\#\left\{(a, x) \in \mathbb{F}_{p}^{2}: F_{a}^{(k)}(x)=0\right\}=p+O\left(4^{k} p^{1 / 2}\right), \quad \text { as } p \rightarrow \infty,
$$

which, together with (4.1), implies the desired result (as we can always assume that $D_{k}>4^{k}$, so $\left.4^{k} p^{1 / 2}>8^{k}\right)$.

In particular, we see from Proposition 4.1 that for any fixed integer $k \geqslant 1$

$$
\mathcal{C}_{k} \sim p / k, \quad \text { as } p \rightarrow \infty .
$$

Note that using [Gao and Rodrigues 2003, Theorem 1] or [Ruppert 1986, Satz B] or [Zannier 1997, Corollary], one can obtain an explicit form for $D_{k}$. However, any such estimate has to depend on the size of the coefficients of $F_{A}^{(k)}(X)$ (considered as a bivariate polynomial in $A$ and $X$ over $\mathbb{Z})$ and is likely to be double exponential in $k$.

We can also compute the exact values of $\mathcal{C}_{1}$ and $\mathcal{C}_{2}$.

Proposition 4.2. For any odd prime $p$, we have $\mathcal{C}_{1}=p$ and $\mathcal{C}_{2}=$ $(p-1) / 2$.

Proof. First, note that any point $x$ contributing to $\mathcal{C}_{1}$ is a root of $F_{a}^{(1)}(X)$ for some $a$, and also

$$
F_{a}^{(1)}(X)=X^{2}-X+a=(X-1 / 2)^{2}+a-1 / 4=0
$$

is solvable if and only if $1 / 4-a$ is a square. Since there are $(p-1) / 2$ squares in $\mathbb{F}_{p}^{*}$, we have $\mathcal{C}_{1}=p$. 
Now, it is easy to see that

$$
F_{a}^{(2)}(X)=X^{2}+X+a+1
$$

If a point $x$ lies in a cycle of length 2 in $\mathcal{G}_{a}$, then it is a root of $F_{a}^{(2)}(X)$ and also it is not a root of $F_{a}^{(1)}(X)$. However, if there exists a point $x$ such that

$$
F_{a}^{(2)}(x)=F_{a}^{(1)}(x)=0,
$$

then we must have $x=-1 / 2, a=-3 / 4$. So, if $a \neq-3 / 4$, then any root of $F_{a}^{(2)}(X)$ lies in a cycle of length 2 . Thus, noticing that

$$
F_{a}^{(2)}(X)=(X+1 / 2)^{2}+a+3 / 4=0
$$

is solvable if and only if $-a-3 / 4$ is a square, we have $\mathcal{C}_{2}=(p-1) / 2$ and conclude the proof.

Table 4.1 shows the $\mathcal{C}_{k}$ for some values of $p$ (in these cases, we

\begin{tabular}{|c|c|c|c|c|c|c|}
\hline \multirow[t]{2}{*}{$k$} & \multicolumn{2}{|c|}{$p=100,003$} & \multicolumn{2}{|c|}{$p=500,009$} & \multicolumn{2}{|c|}{$p=1,000,003$} \\
\hline & $\mathcal{C}_{k}$ & $\lfloor p / k\rfloor$ & $\mathcal{C}_{k}$ & $\lfloor p / k\rfloor$ & $\mathcal{C}_{k}$ & $\lfloor p / k\rfloor$ \\
\hline 1 & 100,003 & 100,003 & 500,009 & 500,009 & $1,000,003$ & $1,000,003$ \\
\hline 2 & 50,001 & 50,001 & 250,004 & 250,004 & 500,001 & 500,001 \\
\hline 3 & 33,333 & 33,334 & 166,669 & 166,669 & 333,333 & 333,334 \\
\hline 4 & 24,890 & 25,000 & 125,000 & 125,002 & 249,890 & 250,000 \\
\hline 5 & 20,061 & 20,000 & 99,353 & 100,001 & 199,310 & 200,000 \\
\hline 6 & 16,775 & 16,667 & 83,664 & 83,334 & 165,852 & 166,667 \\
\hline 7 & 14,179 & 14,286 & 71,582 & 71,429 & 143,109 & 142,857 \\
\hline 8 & 12,474 & 12,500 & 62,541 & 62,501 & 125,266 & 125,000 \\
\hline
\end{tabular}
also included the graphs $X^{2}$ and $\left.X^{2}-2\right)$. This is consistent with Proposition 4.1.

TABLE 4.1. Number of cycles of length $k$

\section{Distribution OF COMPONEnts With Size $k$}

We now study the components of functional graphs by analysing the distribution of their sizes. For the minimal and maximal numbers of components in graphs $\mathcal{G}_{a}$ as well as the popular component size, we refer to [Konyagin et al. 2016, Sections 4.4 and 4.5].

Let $\mathcal{N}_{p}$ be the number of components taken over all $\mathcal{G}_{a}$ modulo $p$, and let $\mathcal{N}_{p, k}$ be the number of those components with size $k>0$ (that is, there are $k$ nodes in the component). Furthermore, let

$$
\mathcal{N}_{p, \text { even }}^{K}=\sum_{\substack{k \leqslant K \\ k \text { even }}} \mathcal{N}_{p, k} \text { and } \quad \mathcal{N}_{p, \text { odd }}^{K}=\sum_{\substack{k \leqslant K \\ k \text { odd }}} \mathcal{N}_{p, k}
$$


Clearly,

We first have:

$$
\mathcal{N}_{p}=\mathcal{N}_{p, \text { even }}^{p}+\mathcal{N}_{p, \text { odd }}^{p}
$$

Proposition 5.1. For any odd prime $p, \mathcal{N}_{p, 2}=(p-1) / 2$.

Proof. If $C$ is a component of $\mathcal{G}_{a}$ of size 2 , then it is easy to see that $C=\{x,-x\}$ for some $x \in \mathbb{F}_{p}$ such that $x$ is a fixed point (that is, $x^{2}+a=x$ ) and the equation $X^{2}+a=-x$ has no solution in $\mathbb{F}_{p}$ (that is, $-x-a$ is not a square).

In other words, for any $x \in \mathbb{F}_{p}$, if we choose $a=-x^{2}+x$, then $x$ is a fixed point in $\mathcal{G}_{a}$ and $-x-a=x^{2}-2 x$. So, it is equivalent to count how many $x \in \mathbb{F}_{p}$ such that $x^{2}-2 x$ is not a square in $\mathbb{F}_{p}$. Since $x^{2}-2 x=(x-1)^{2}-1$, it is also equivalent to count how many $x \in \mathbb{F}_{p}$ such that $x^{2}-1$ is not a square in $\mathbb{F}_{p}$.

If $x^{2}-1$ is a square in $\mathbb{F}_{p}$, say $x^{2}-1=y^{2}$, then we have $(x+y)(x-$ $y)=1$. Let $\alpha=x+y$, then $x-y=\alpha^{-1}$, and so

$$
x=\frac{\alpha+\alpha^{-1}}{2}, \quad y=\frac{\alpha-\alpha^{-1}}{2} .
$$

So, for such pairs $(x, y)$ we obtain a one-to-one correspondence between pairs $(x, y)$ and pairs $\left(\alpha, \alpha^{-1}\right), \alpha \neq 0$. It is easy to see that for any $\alpha_{1}, \alpha_{2} \in \mathbb{F}_{p}^{*}$,

$$
\frac{\alpha_{1}+\alpha_{1}^{-1}}{2}=\frac{\alpha_{2}+\alpha_{2}^{-1}}{2} \text { if and only if } \alpha_{1} \alpha_{2}=1 .
$$

So, by counting the pairs $\left(\alpha, \alpha^{-1}\right)$, there are $(p+1) / 2$ values of $x$ such that $x^{2}-1$ is a square. Therefore, there are $(p-1) / 2$ values of $x$ such that $x^{2}-1$ is not a square. This completes the proof.

It has been predicted in [Flajolet and Odlyzko 1990, Theorem 2 (i)] that

$$
\mathcal{N}_{p} \sim \frac{p \log p}{2}
$$

which has a small bias (about 9.5\%) over the real value; see [Konyagin et al. 2016, Table 4.2]. Here, we improve the precision of this estimate. First, we note that each node in $\mathcal{G}_{a}$ has in-degree two or zero except for the node $a$, since only 0 maps to $a$. Therefore, each component in any graph $\mathcal{G}_{a}$ has an even number of nodes unless it is the component containing 0 and $a$. So, each graph $\mathcal{G}_{a}$ has exactly one component of odd size. It follows that

$$
\mathcal{N}_{p, \text { odd }}^{p}=p
$$

and so

$$
\mathcal{N}_{p} \sim \mathcal{N}_{p, \text { even }}^{p}, \quad \text { as } p \rightarrow \infty
$$


For even-sized components, the situation is not as straightforward. In our experiments, we noticed that the number of even-sized components with size $k$ is very close to $p / k$ as shown in Table 5.1 for $k \leqslant 20$ and for $k=1000$ and 2000 (i.e., even for larger values of $k$ ).

\begin{tabular}{rrrrrrr}
\hline$k$ & \multicolumn{2}{c}{$p=100,003$} & \multicolumn{2}{c}{$p=500,009$} & \multicolumn{2}{c}{$p=1,000,003$} \\
& $\mathcal{N}_{p, k}$ & $\lfloor p / k\rfloor$ & $\mathcal{N}_{p, k}$ & $\lfloor p / k\rfloor$ & $\mathcal{N}_{p, k}$ & \multicolumn{1}{c}{$\lfloor/ k\rfloor$} \\
\hline 2 & 50,001 & 50,001 & 250,004 & 250,004 & 500,001 & 500,001 \\
4 & 24,951 & 25,000 & 125,160 & 125,002 & 250,171 & 250,000 \\
6 & 16,156 & 16,667 & 83,185 & 83,334 & 166,660 & 166,667 \\
8 & 12,509 & 12,500 & 62,652 & 62,501 & 124,727 & 125,000 \\
10 & 10,083 & 10,000 & 50,422 & 50,000 & 99,975 & 100,000 \\
12 & 8,389 & 8,333 & 41,542 & 41,667 & 82,577 & 83,333 \\
14 & 7,192 & 7,143 & 35,661 & 35,714 & 71,611 & 71,428 \\
16 & 6,292 & 6,250 & 31,186 & 31,350 & 62,220 & 62,500 \\
18 & 5,503 & 5,555 & 27,941 & 27,778 & 55,923 & 55,555 \\
20 & 5,009 & 5,000 & 24,662 & 25,000 & 50,135 & 50,000 \\
1000 & 117 & 100 & 533 & 500 & 954 & 1,000 \\
2000 & 48 & 50 & 243 & 250 & 489 & 500 \\
\hline \multicolumn{7}{c}{}
\end{tabular}

TABLE 5.1. Number of components of size $k$

Now, using $\lfloor p / k\rfloor$ as an approximation of the number of components of size $k$ for any even $k<p$, we can get an approximation for $\mathcal{N}_{p \text {,even }}^{p}$. First, when $(p-1) / 2<k<p$, we have $\lfloor p / k\rfloor=1$, and there are about $(p-1) / 4$ values of such even $k$. In general, if $(p-1) /(n+1)<k \leqslant$ $(p-1) / n$, we have $\lfloor p / k\rfloor=n$, and there are about $\frac{p-1}{2 n(n+1)}$ values of such even $k$, which contributes to around $\frac{p-1}{2(n+1)}$ components of even size.

Fixing a positive integer $n$, for $k>(p-1) /(n+1)$ we use the above estimate, while for $k \leqslant(p-1) /(n+1)$ we use the estimate $(p-1) / k$, and so the total number of components of even size is around

$$
\begin{aligned}
\frac{p-1}{2}\left(1+\frac{1}{2}+\right. & \left.\cdots+\frac{1}{(p-1) /(2(n+1))}\right) \\
& +\frac{p-1}{2}\left(\frac{1}{2}+\frac{1}{3}+\cdots+\frac{1}{n+1}\right)
\end{aligned}
$$


which, together with the approximation of the harmonic series, is approximated by

$$
\begin{aligned}
\frac{p-1}{2}\left(\log \frac{p-1}{2(n+1)}+\gamma\right) & +\frac{p-1}{2}(-1+\log (n+1)+\gamma) \\
= & \frac{p-1}{2}(\log (p-1)+2 \gamma-1-\log 2),
\end{aligned}
$$

where $\gamma=0.5772156649 \ldots$ is the Euler constant. So, we denote

$$
\tilde{\mathcal{N}}_{p, \text { even }}^{p}=\frac{p-1}{2}(\log (p-1)+2 \gamma-1-\log 2),
$$

which is an approximation of $\mathcal{N}_{p \text {,even }}^{p}$.

Table 5.2 shows the difference between the two values for several large primes. We overestimate the actual value by about $2 \%$.

\begin{tabular}{rcccc}
\hline \multicolumn{1}{c}{$p$} & $\mathcal{N}_{p, \text { even }}^{K}$ & $\mathcal{N}_{p \text {,even }}^{p}$ & $\mathcal{N}_{p}$ & $\tilde{\mathcal{N}}_{p, \text { even }}^{p}$ \\
\hline 100,003 & 521,337 & 538,640 & 638,643 & 548,722 \\
200,003 & $1,113,083$ & $1,147,694$ & $1,347,697$ & $1,166,748$ \\
300,007 & $1,730,420$ & $1,782,805$ & $2,082,812$ & $1,810,962$ \\
400,009 & $2,364,734$ & $2,434,894$ & $2,834,903$ & $2,472,154$ \\
500,009 & $3,011,626$ & $3,098,914$ & $3,598,923$ & $3,145,966$ \\
600,011 & $3,667,637$ & $3,772,277$ & $4,372,288$ & $3,829,859$ \\
700,001 & $4,333,622$ & $4,455,913$ & $5,155,914$ & $4,522,041$ \\
800,011 & $5,005,995$ & $5,145,194$ & $5,945,205$ & $5,221,530$ \\
900,001 & $5,685,731$ & $5,842,337$ & $6,742,338$ & $5,927,145$ \\
$1,000,003$ & $6,369,257$ & $6,543,317$ & $7,543,320$ & $6,638,411$ \\
\hline
\end{tabular}

TABLE 5.2. Estimates for the number of components with even size and $K=(p-1) / 2$

\section{Shape of TREes in FUnCtional GRAPHS}

Finally, in order to reveal more detailed features of functional graphs, we consider the trees attached to such graphs.

In the functional graph $\mathcal{G}_{a}$ corresponding to $f_{a}$, each node in a cycle, except for $a$ (if $a$ lies in a cycle), is connected to a unique node (say $w$ ) which is not in the cycle. Naturally, we treat the node $w$ as the root of the binary tree attached to a cyclic point in the graph $\mathcal{G}_{a}$. Thus, we can say that each node in a cycle of $\mathcal{G}_{a}$, expect for $a$, is associated with a binary tree - in fact a full binary tree, unless 0 is a node in the tree. For example, in Figure 1.1, there are 8 full binary trees attached 
to the cyclic points. Let $t_{p}(a, k)$ be the number of such binary trees with $k$ nodes in $\mathcal{G}_{a}$, and let

$$
T_{p}(k)=\sum_{a \in \mathbb{F}_{p}} t_{p}(a, k) \quad \text { and } \quad T_{p}=\sum_{k=1}^{p-1} T_{p}(k)
$$

and for the connected graphs equivalents, let

$$
T_{p}^{*}(k)=\sum_{a \in \mathcal{I}_{p}} t_{p}(a, k) \quad \text { and } \quad T_{p}^{*}=\sum_{k=1}^{p-1} T_{p}^{*}(k) .
$$

Note that $T_{p}$ is the total number of trees attached to all such functional graphs $\mathcal{G}_{a}$, and $T_{p}^{*}$ has a similar meaning but with restriction to connected functional graphs.

An interesting question is whether these trees behave similarly to random full binary trees. First we observe that there is a significant proportion of trees with just one node, as shown in Table 6.1 for the general case and in Table 6.2 for connected graphs. This motivates us to pose the following conjecture, which seems to be reasonable because exactly half of elements in $\mathbb{F}_{p}^{*}$ are not square.

Conjecture 6.1. We have $T_{p}(1) / T_{p} \sim 1 / 2$ as $p \rightarrow \infty$.

\begin{tabular}{rrrr}
\hline \multicolumn{1}{c}{$p$} & \multicolumn{1}{c}{$T_{p}(1)$} & \multicolumn{1}{c}{$T_{p}$} & $\%$ \\
\hline 50,111 & $7,090,084$ & $14,091,820$ & $50.31 \%$ \\
100,003 & $19,845,915$ & $39,530,737$ & $50.20 \%$ \\
200,003 & $56,210,936$ & $112,088,213$ & $50.15 \%$ \\
300,007 & $103,203,596$ & $205,901,181$ & $50.12 \%$ \\
400,009 & $158,746,944$ & $317,089,081$ & $50.06 \%$ \\
500,009 & $221,941,725$ & $443,336,032$ & $50.06 \%$ \\
$1,000,003$ & $627,460,216$ & $1,253,326,817$ & $50.06 \%$ \\
\hline
\end{tabular}

TABLE 6.1. Number of trees with one node 


\begin{tabular}{rrrr}
\hline \multicolumn{1}{c}{$p$} & \multicolumn{1}{c}{$T_{p}^{*}(1)$} & \multicolumn{1}{c}{$T_{p}^{*}$} & $\%$ \\
\hline 50,111 & 27,877 & 55,668 & $50.08 \%$ \\
100,003 & 52,923 & 105,612 & $50.11 \%$ \\
200,003 & 115,746 & 231,583 & $49.98 \%$ \\
300,007 & 161,975 & 323,410 & $50.08 \%$ \\
400,009 & 222,865 & 445,931 & $49.98 \%$ \\
500,009 & 298,060 & 595,142 & $50.08 \%$ \\
$1,000,003$ & 542,592 & $1,086,147$ & $49.96 \%$ \\
\hline
\end{tabular}

TABLE 6.2. Number of trees with one node in connected graphs

Second, for large trees, we check the average height of the trees in the graphs. It has been shown in [Flajolet and Odlyzko 1982, Theorem B] that the average height of full binary trees with $n$ internal nodes is

$$
\bar{H}_{n} \sim 2 \sqrt{\pi n} \quad \text { as } n \rightarrow \infty .
$$

This means that for a random full binary tree, its height is asymptotic to $2 \sqrt{\pi n}$ when $n$ goes to the infinity. In our situation, for each tree with $n$ internal nodes and height $H_{n}$, we compute the ratio $H_{n} / 2 \sqrt{\pi n}$ and find the average of this ratio for all graphs modulo $p$. (Again, a tree is not always guaranteed to be a full binary tree, since 0 might be a node in the tree, but the impact of this happening is negligible, and at any case, we collect trees of both sizes $2 n$ and $2 n+1$.)

In Table 6.3, we compare the ratio of $\bar{H}_{n} / 2 \sqrt{\pi n}$ (see [Flajolet and Odlyzko 1982, Table II]) with the average ratio of $H_{n} / 2 \sqrt{\pi n}$ of the trees in our graphs. One can see that they are close.

\begin{tabular}{rrrrr}
\hline \multicolumn{1}{c}{$n$} & $\bar{H}_{n} / 2 \sqrt{\pi n}$ & \multicolumn{3}{c}{ average of $H_{n} / 2 \sqrt{\pi n}$} \\
& & $p=50111$ & $p=100003$ & $p=200003$ \\
\hline 50 & 0.797 & 0.837 & 0.837 & 0.837 \\
100 & 0.846 & 0.875 & 0.873 & 0.872 \\
500 & 0.920 & 0.952 & 0.925 & 0.941 \\
1,000 & 0.940 & 0.925 & 0.948 & 0.942 \\
2,000 & 0.956 & 0.981 & 0.944 & 0.960 \\
5,000 & 0.970 & 0.927 & 0.916 & 0.977 \\
\hline
\end{tabular}

TABle 6.3. Average height of trees 


\section{Future Directions}

One of the most important directions in this area is developing an adequate random model predicting the statistical characteristics of the functional graphs of polynomials, see [Martin and Panario 2016] for some initial, yet promising results in this direction.

Based on our computations, we pose several conjectures about the functional graphs of quadratic polynomials. Investigating whether they are true or not may help to characterise functional graphs generated by quadratic polynomials and understand the similarities and differences between these functional graphs and random mappings.

The other interesting problem is to count the number of functional graphs modulo $p$ generated by quadratic polynomials up to isomorphism; see [Konyagin et al. 2016, Theorem 2.8] for a lower bound. In [Gilbert et al. 2001, Conjecture C] the authors conjectured that for any odd prime $p \neq 17$, there are $p$ such functional graphs up to isomorphism, and they confirmed this for all the odd primes up to 1009 not equal to 17 . Under our computations, we confirm this conjecture for all the odd primes up to 100000 not equal to 17.

\section{ACKNOWLEDGEMENTS}

The authors are grateful to Patric Morton and Michael Zieve for several useful suggestions and literature references, especially concerning dynatomic polynomials.

For the research, B.M. was partially supported by the Australian Research Council Grants DP140100118 and DP170102794, M.S. by the Macquarie University Research Fellowship, I.S. by the Australian Research Council Grants DP130100237 and DP140100118.

\section{REFERENCES}

[Bellah et al. 2016] E. Bellah, D. Garton, E. Tannenbaum and N. Walton, 'A probabilistic heuristic for counting components of functional graphs of polynomials over finite fields', Preprint, 2016 (see http://arxiv.org/abs/1609.07667). (p. 1)

[Bridy and Garton 2016] A. Bridy and D. Garton, 'Dynamically distinguishing polynomials', Preprint, 2016 (see http://arxiv.org/abs/1609.09186). (p. 1)

[Burnette and Schmutz 2017] C. Burnette and E. Schmutz, 'Periods of iterated rational functions over a finite field', Intern. J. Number Theory, 13 (2017), no.5, 1301-1315. (p. 1)

[Flajolet and Odlyzko 1982] P. Flajolet, A. Odlyzko, 'The average height of binary trees and other simple trees', J. Comput. System Sci., 25 (1982), no. 2, 171-213. (p. 20) 
[Flajolet and Odlyzko 1990] P. Flajolet, A. Odlyzko, 'Random mapping statistics', Lecture Notes in Computer Science, 434(1990), 329-354. (pp. 1, 3, 8, and 16)

[Flynn and Garton 2014] R. Flynn and D. Garton, 'Graph components and dynamics over finite fields', Intern. J. Number Theory, 10 (2014), 779-792. (p. 1)

[Gao and Rodrigues 2003] S. Gao and V. M. Rodrigues, 'Irreducibility of polynomials modulo p via Newton polytopes' J. Number Theory, 101 (2003), 32-47. (p. 14)

[Gilbert et al. 2001] C. L. Gilbert, J. D. Kolesar, C. A. Reiter and J. D. Storey, 'Function digraphs of quadratic maps modulo p', Fibonacci Quart., 39 (2001), 32-49. (p. 21)

[Konyagin et al. 2016] S. V. Konyagin, F. Luca, B. Mans, L. Mathieson, M. Sha and I. E. Shparlinski, 'Functional graphs of polynomials over finite fields', J. Combin. Theory, Ser. B, 116 ( 2016), 87-122. (pp. 1, 2, 3, 4, 8, 15, 16, and 21)

[Lorenzini 1996] D. Lorenzini, An invitation to arithmetic geometry, Amer. Math. Soc., 1996. (p. 14)

[Martin and Panario 2016] R. S. V. Martin and D. Panario, 'On the heuristic of approximating polynomials over finite fields by random mappings', Intern. J. Number Theory, 12 (2016), 1987-2016. (p. 21)

[Morton 1996] P. Morton, 'On certain algebraic curves related to polynomial maps', Compositio Math., 103 (1996), 319-350; Corrigendum, Compositio Math., 147 (2011), 332-334. (p. 14)

[Morton and Patel 1994] P. Morton and P. Patel, 'The Galois theory of periodic points of polynomial maps', Proc. London Math. Soc., 68 (1994), 225-263. (p. 13)

[Morton and Vivaldi 1995] P. Morton and F. Vivaldi, 'Bifurcations and Discriminants for Polynomial Maps', Nonlinearity 8 (1995), 571-584. (p. 14)

[NTL 2016] NTL: A Library for doing Number Theory, 2016, http://shoup.net/ntl/. (p. 6)

[Ostafe and Sha 2016] A. Ostafe and M. Sha, 'Counting dynamical systems over finite fields', Contemp. Math., v.669, Amer. Math. Soc., 2016, 187-203. (p. 1)

[Pari 2016] The PARI Group, PARI/GP version 2.9.0, Univ. Bordeaux, 2016, http://pari.math.u-bordeaux.fr/. (p.6)

[Peinado et al. 2001] A. Peinado, F. Montoya, J. Muñoz and A. J. Yuste, 'Maximal periods of $x^{2}+c$ in $\mathbb{F}_{q}$ ', in S. Boztaş and I. E. Shparlinski (Eds.): Applied Algebra, Algebraic Algorithms and Error-Correcting Codes, Lecture Notes in Comput. Sci. 2227, Springer, Berlin, 2001, pp. 219-228. (p. 13)

[Ruppert 1986] W. M. Ruppert, 'Reduzibilität ebener kurven', J. Reine Angew. Math., 369 (1986), 167-191. (p. 14)

[Silverman 2007] J. H. Silverman, The arithmetic of dynamical systems, Springer Verlag, 2007. (p. 4)

[Vasiga and Shallit 2004] T. Vasiga and J. O. Shallit, 'On the iteration of certain quadratic maps over GF(p)', Discr. Math., 277 (2004), 219-240. (p. 3)

[Zannier 1997] U. Zannier, 'On the reduction modulo $p$ of an absolutely irreducible polynomial $f(x, y)$ ', Arch. Math., 68 (1997), 129-138. (p. 14) 
B.M.: Department of Computing, Macquarie University, Sydney, NSW 2109, Australia

E-mail address: bernard.mans@mq.edu.au

M.S.: Department of Computing, Macquarie University, Sydney, NSW 2109, Australia

E-mail address: shamin2010@gmail.com

I.S.: Department of Pure Mathematics, University of New South Wales, Sydney, NSW 2052, Australia

E-mail address: igor.shparlinski@unsw.edu.au

D.S.: Department of Computing, Macquarie University, Sydney, NSW 2109, Australia

E-mail address: daniel.sutantyo@gmail.com 